\title{
BMJ Open Protection levels of N95-level respirator substitutes proposed during the COVID-19 pandemic: safety concerns and quantitative evaluation procedures
}

\author{
David H Ballard (D , ${ }^{1}$ Audrey J Dang, ${ }^{2}$ Benjamin M Kumfer, ${ }^{2}$ Patricia B Weisensee, ${ }^{3}$ \\ J Mark Meacham, ${ }^{3}$ Alex R Scott, ${ }^{4}$ Mary Ruppert-Stroescu, ${ }^{5}$ Broc A Burke, ${ }^{6}$ \\ Jason Morris, ${ }^{4}$ Connie Gan, ${ }^{4}$ Jesse Hu, ${ }^{4}$ Bradley King, ${ }^{7}$ \\ Udayabhanu Jammalamadaka, ${ }^{1}$ Sena Sayood, ${ }^{8}$ Stephen Liang, ${ }^{8}$ \\ Shruti Choudhary, ${ }^{2}$ David Dhanraj, ${ }^{2}$ Bruno Maranhao, ${ }^{6}$ Christine Millar, ${ }^{9}$ \\ J Tyler Bertroche, ${ }^{10}$ Nirah Shomer, ${ }^{11}$ Pamela K Woodard, ${ }^{1}$ Pratim Biswas, ${ }^{2}$ \\ Richard Axelbaum, ${ }^{2}$ Guy Genin, ${ }^{3,12,13}$ Brent J Williams, ${ }^{2}$ Kathleen Meacham ${ }^{6}$
}

To cite: Ballard DH, Dang AJ, Kumfer BM, et al. Protection levels of N95level respirator substitutes proposed during the COVID-19 pandemic: safety concerns and quantitative evaluation procedures. BMJ Open 2021;11:e045557. doi:10.1136/ bmjopen-2020-045557

- Prepublication history and additional supplemental material for this paper are available online. To view these files, please visit the journal online (http://dx.doi.org/10.1136/ bmjopen-2020-045557).

Received 06 October 2020 Accepted 03 August 2021

Check for updates

(c) Author(s) (or their employer(s)) 2021. Re-use permitted under CC BY-NC. No commercial re-use. See rights and permissions. Published by BMJ.

For numbered affiliations see end of article.

Correspondence to Dr Kathleen Meacham; meachamk@wustl.edu

\section{ABSTRACT}

Objective The COVID-19 pandemic has precipitated widespread shortages of filtering facepiece respirators (FFRs) and the creation and sharing of proposed substitutes (novel designs, repurposed materials) with limited testing against regulatory standards. We aimed to categorically test the efficacy and fit of potential N95 respirator substitutes using protocols that can be replicated in university laboratories.

Setting Academic medical centre with occupational health-supervised fit testing along with laboratory studies. Participants Seven adult volunteers who passed quantitative fit testing for small-sized $(n=2)$ and regularsized $(n=5)$ commercial $N 95$ respirators.

Methods Five open-source potential N95 respirator substitutes were evaluated and compared with commercial National Institute for Occupational Safety and Health (NIOSH)-approved N95 respirators as controls. Fit testing using the 7-minute standardised 0ccupational Safety and Health Administration fit test was performed. In addition, protocols that can be performed in university laboratories for materials testing (filtration efficiency, air resistance and fluid resistance) were developed to evaluate alternate filtration materials.

Results Among five open-source, improvised substitutes evaluated in this study, only one (which included a commercial elastomeric mask and commercial HEPA filter) passed a standard quantitative fit test. The four alternative materials evaluated for filtration efficiency $(67 \%-89 \%)$ failed to meet the $95 \%$ threshold at a face velocity $(7.6 \mathrm{~cm} / \mathrm{s})$ equivalent to that of a NIOSH particle filtration test for the control N95 FFR. In addition, for all but one material, the small surface area of two 3D-printed substitutes resulted in air resistance that was above the maximum in the NIOSH standard.

Conclusions Testing protocols such as those described here are essential to evaluate proposed improvised respiratory protection substitutes, and our testing platform could be replicated by teams with similar crossdisciplinary research capacity. Healthcare professionals
Strengths and limitations of this study

Manufacturing of open-source potential N95 respirator substitutes, quantitative fit testing, filtration testing and materials testing reflecting a method for others in a university lab setting to test N95 proposed substitute for a pandemic-related response.

- Quantitative fit testing according to Occupational Safety and Health Administration provides an objective measure of how the N95 alternative substitutes perform on individuals who passed fit testing on commercial $\mathrm{N} 95$ respirators.

- Filtration data give performance of improvised filter materials and how they perform at velocities relevant to normal breathing and filtering in the range of SARS-CoV-2 viral particles.

- Limitation of the production of these open-source substitutes was produced to the best of the authors' understanding of posted instructions and did not attempt proposed substitutes to improve the mask designs.

should be cautious of claims associated with improvised respirators when suggested as FFR substitutes.

\section{INTRODUCTION}

Personal protective equipment (PPE) is critical for limiting infectious disease risk to clinicians. During the COVID-19 pandemic, the WHO noted in February 2020 that the global stockpile of PPE was insufficient, particularly for masks and filtering facepiece respirators (FFRs). ${ }^{1}$ In a survey in March 2020 by the Association for Professionals in Infection Control and Epidemiology, nearly half of respondents reported that their healthcare facility's N95 FFR supply was nearly or 

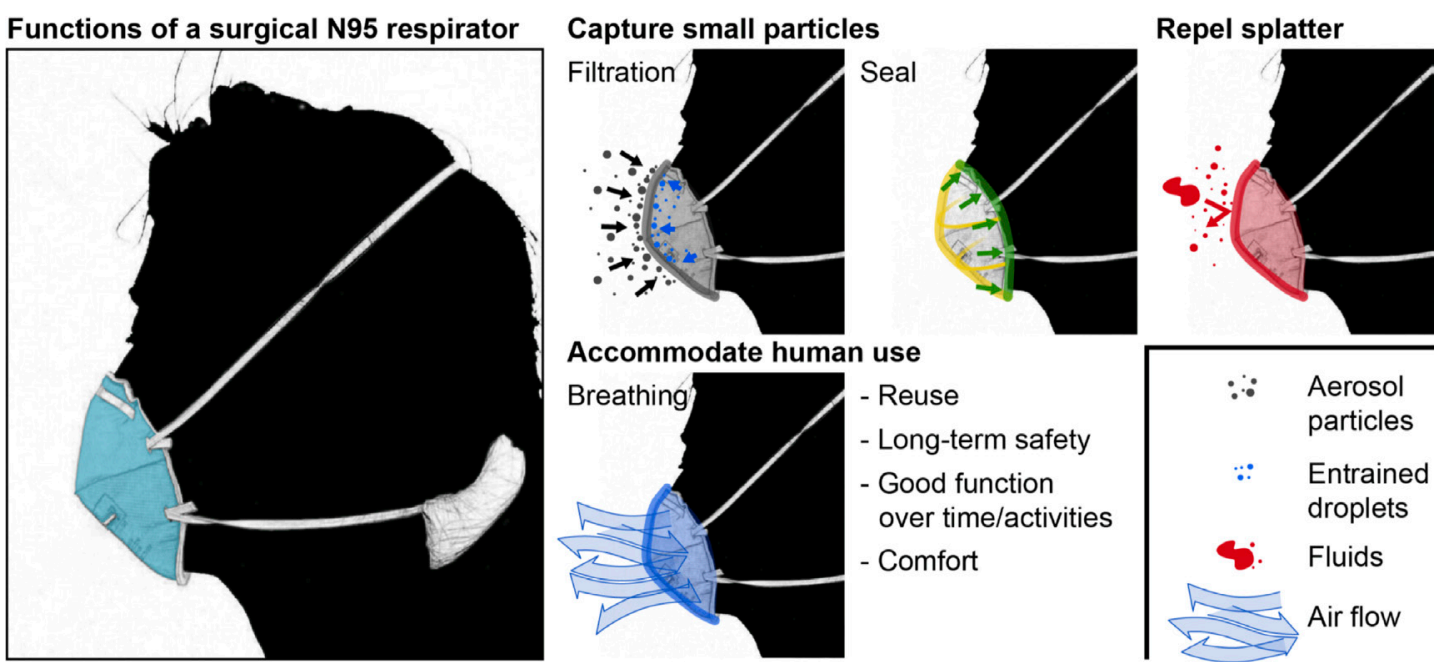

Figure 1 Overview of essential surgical N95 attributes.

completely depleted. ${ }^{2}$ To address these shortages, many institutions developed alternatives to commercial FFRs to provide immediate stopgap solutions. ${ }^{2-11}$ Some of these proposed substitutes were publicly disseminated, often with limited testing of key attributes including filtration, breathability, fit and liquid fluid repellency.

\section{Key functional attributes of N95 FFRs}

In the USA, surgical N95 FFRs used by healthcare personnel are regulated by both the National Institute for Occupational Safety and Health (NIOSH) and the Food and Drug Administration. The surgical N95 respirator serves to protect wearers by filtering fine particles, providing a tight seal around the face, and repelling fluid splatter, while ensuring ease of breathing (figure 1). ${ }^{12} 13$ Particle filtration efficiency is dependent on the size of the particle, the material properties of the respirator and the face velocity at which the particle approaches the material; the face velocity depends on the user's instantaneous respiratory rate and the shape and size of the respirator itself. Respirator form must ensure that all breathed air passes through the filtration medium and does not leak from an edge. Lower flow resistance (larger surface area, material with lower pressure drop) reduces the work of breathing, mitigating wearer fatigue. The respirator must be comfortable, and respirator materials cannot pose health risks to the wearer (ie, should not shed hazardous particles or fibres that can be inhaled). During crises, the respirator may need to function over periods of extended use and be reused; therefore, the respirator should be suitable for sterilisation and maintain structural integrity. More specifically, supply of commercial N95 respirators has been conserved during the COVID-19 pandemic by multiple sterilisation methods including hydrogen peroxide vapour, chlorine dioxide vapour, steam, ultraviolet radiation, heat and isolation over time ${ }^{14-16}$ Finally, in the patient care environment, the filter material and/or an outer covering should repel high-velocity fluid splatter.

Due to the critical shortage of N95 respirators during the early COVID-19 pandemic, many institutions resorted to using locally improvised masks which have not undergone appropriate safety testing. As such, a discrepancy may exist between the respiratory protection actually provided by an improvised design and that the level of protection which healthcare workers would expect of a commercial respirator. Testing recently developed, opensource designs intended as proposed substitutes for N95 respirators, we present our framework of establishing an institutional platform for evaluating these improvised designs and materials, including fit, filtration and fluid repellency testing. This framework could be replicated by collaborative teams with similar cross-disciplinary expertise and laboratory capabilities.

\section{METHODS}

\section{Overview}

Five open-source, improvised respirator designs were selected for testing based on their wide public dissemination (during the early COVID-19 pandemic, March-April 2020) in order to demonstrate testing procedures and identify efficacy and potential limitations (figure 2): a cloth-based respirator ('Sewn Sterilization Wrap'), ${ }^{7}$ three 3D-printed respirators ('P100 Adaptor', 'Self-Moldable 3D Printed' ${ }^{, 9}$ and 'Multi-Part 3D Printed ${ }^{10}$ ) and one repurposed from medical supplies ('Elastomeric'). ${ }^{11}$ These were produced as detailed in online supplemental data document. A commercial NIOSH-approved N95 respirator (disposable 3M 1860 Health Care Particulate N95 FFR Respirators, 3M, St Paul, Minnesota, USA) served as control. Experiments were performed in laboratories at our institution. Testing included Occupational Safety and Health Administration (OSHA)-standard quantitative fit testing, filtration testing in an aerosol laboratory and liquid repellency testing in a surface chemistry laboratory.

Several of these designs could be fabricated using different filtration media, and we evaluated several candidates that have been proposed for use in these opensource designs. Filtration efficiency and liquid repellency 


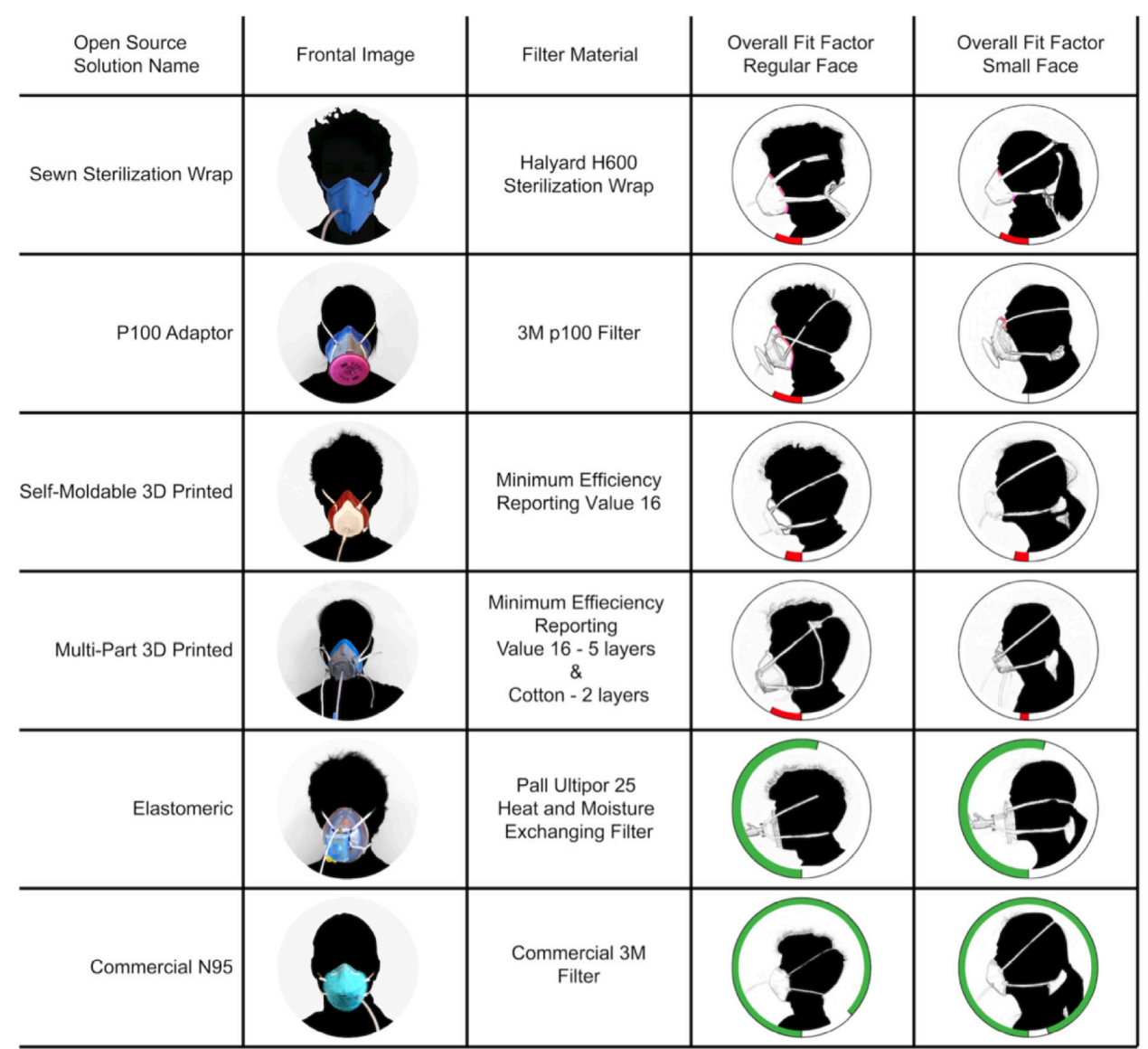

Figure 2 The six designs are displayed with an image of them on a user in the second column, and the filter material used in the third column. The last two columns present the respirators stratified by standardised face size of the user. Radial bar plots display overall fit factor from the OSHA 7-minute standardised fit test for each design as well as the 3M N95 for regular and small size standardised users. Green bars represent passing scores, 100 or greater, while red bars indicate failing scores. Areas noted by users to leak air were highlighted. OSHA, Occupational Safety and Health Administration.

were evaluated for Halyard H600 sterilisation wrap (O\&M Halyard, Alpharetta, Georgia, USA) and Filti Face Mask Material (Filti, Lenexa, Kansas, USA). In addition, filtration efficiency was also evaluated for a second Halyard sterilisation wrap (H500, O\&M Halyard, Alpharetta, Georgia, USA), material from a commercial N95 respirator (3M VFlex Healthcare Particulate Respirator and Surgical Mask 1804, 3M, St Paul, Minnesota, USA), and commercial HVAC material (MERV16 rating), and other configurations of the sterilisation wrap materials (two layers of $\mathrm{H} 600$, single layers of $\mathrm{H} 600$ with stitching).

\section{Patient and public involvement}

The authors (including those who originated the study) and fit testing volunteers include intended users (ie, healthcare workers) of the improvised respirator designs studied in this work. No patients were involved in this research.

\section{Quantitative respiratory fit testing}

Respirators were quantitatively tested via OSHA 7-minute standardised fit test ${ }^{17}$ using a PortaCount Respirator Fit Tester Model 8048 and TSI Model 8026 Particle Generator with TSI FitPro Ultra software. A $4 \mathrm{~mm}$ metal grommet was punched through each respirator at a location not in direct contact with skin and connected with $4 \mathrm{~mm}$ tubing to the PortaCount device. To facilitate testing of 3D-printed respirators, the grommet was inserted through the filter material. To permit passage of a grommet into the filter of the Multi-Part 3D Printed respirator, a soldering iron was used to create a hole in the thermoplastic cap overlying filtration material. Three adult volunteers served as standard faces (two regular, one small). The Self-Moldable 3D Printed respirator was moulded using hot water as described in design instructions (online supplemental data document). Each user adjusted respirator placement and strap tightness during real-time fit testing to achieve the best possible fit prior to the 7-minute OSHA standard test. Each design was tested on faces calibrated to small-sized and regular-sized surgical N95 FFRs.

\section{Materials testing: filtration and breathability}

Particle filtration performance was evaluated for several materials including commercial filtration materials and fabrics intended for other medical uses. Additional information about testing procedures and a sampling diagram 
can be found in online supplemental data document, figure 1. Sample discs of $47 \mathrm{~mm}$ were cut directly from the mask or the sourced material sheet and placed in an inline filter holder during filtration testing (online supplemental data document, figure 2). A polydisperse $\mathrm{NaCl}$ aerosol was produced using a Collison nebuliser, dried to remove water content, and then passed through a charge neutraliser and an electrostatic classifier (TSI, Model 3080 with long differential mobility analyser column), which selected particles based on their mobility in the electric field with a single-charge diameter setpoint of $300 \mathrm{~nm}$ (online supplemental data document for additional discussion of the particle size). The size-classified aerosol was then charge-neutralised a second time and diluted using HEPA-filtered air to achieve a final particle number concentration in the range of 3000-4000 \#/ cc. As per our intention to evaluate how these improvised designs compare with the N95 respirators in short supply, this selected size is consistent with similar filtration studies of $\mathrm{N} 95$ respirators. ${ }^{18}$ Though this diameter is somewhat larger than the size of an isolated SARS-CoV-2 viral particle (approximately $75-105 \mathrm{~nm}$ ), the virus would most likely be in a larger respiratory particle consisting primarily of water, proteins, salts and surfactants. ${ }^{19} 20$

To determine filtration efficiency, particle concentrations upstream and downstream of the filter were measured via continuous condensation particle counter (TSI, Model 3022A). Concentrations were measured in immediate succession to mitigate impact of drift in nebuliser output over time. The NIOSH N95 protocol demands a flow of $85 \mathrm{~L} / \mathrm{min}$ through the entire respirator, reported to yield a face velocity in the range of 10-13 $\mathrm{cm} / \mathrm{s}$ for surface areas typical of commercial N95 respirators. ${ }^{21}$ We report results here for tests at $7.6 \pm 0.1 \mathrm{~cm} / \mathrm{s}$, based on the calculated face velocity for the N95 FFR in this study. Particle filtration efficiency values reported here are the average of the three to four different filter punches for the same material. Methods for these calculations are included in online supplemental data document. The pressure drop across the filter material along with the temperature and relative humidity of the gas passed through the filter was recorded.

\section{Materials testing: liquid repellency and splatter}

Liquid repellency of two of the fabrics used in the alternative respirator designs, Halyard $\mathrm{H} 600$ and Filti, was tested through contact angle and fluid penetration measurements. Advancing and receding contact angles were measured by slowly increasing and decreasing the volume of a sessile droplet using a 30-gauge needle and analysed using ImageJ. ${ }^{22}$ Textile liquid absorbency was evaluated via AATCC (American Association of Textile Chemists and Colorists) test method 79-2018. ${ }^{23}$ Blood splatter testing followed ASTM F1862 ('Resistance of Medical Face Masks to Penetration by Synthetic Blood') procedures, with the following exceptions: (1) room temperature whole milk, dyed with red food colouring, replaced the synthetic blood. The surface tension $\gamma_{1}=49.7 \pm 2.0$
$\mathrm{mN} / \mathrm{m}$ was determined using the pendant drop method with a 16-gauge needle, and was independent of the dye concentration. ${ }^{24}$ (2) Fabrics were typically not preconditioned at $85 \%$ relative humidity $(\mathrm{RH})$. Instead, most were stored in a regular laboratory environment $(35 \%-55 \%$ $\mathrm{RH}, 22^{\circ} \mathrm{C} \pm 1^{\circ} \mathrm{C}$ ). (3) Only a limited number of tests (one to three tests) were performed for each impact velocity and fabric. (4) Pressure levels to achieve the required liquid impact velocities $(4.5,5.5$ and $6.35 \mathrm{~m} / \mathrm{s}$; experimental uncertainty of $\pm 0.07 \mathrm{~m} / \mathrm{s}$ ) were approximately 34 , 50 and $65 \mathrm{kPa}$, respectively, and were calibrated prior to every test session.

\section{RESULTS \\ Quantitative respirator fit testing}

All but one potential N95 respirator substitute evaluated failed to reach the OSHA half-mask respirator overall fit factor minimum of 100; only the Elastomeric substitute (which uses a commercial HEPA filter for particle filtration mounted to a commercial anaesthesia face mask) passed quantitative fit on both small and large face standardised users. Common points of fit failure between respirators were air leak around the nose and difficulty with strap tightening. For 3D-printed respirators, users experienced discomfort due to respirator contact at the chin and bridge of the nose. Individual fit factors and points of failure are noted in figure 2 and online supplemental data document. Components of the quantitative fit test for each potential N95 respirator substitute are noted in figure 3.

The Sewn Sterilization Wrap design failed to reach OSHA specifications (fit factor $>100$ ) for both small and regular respirator size (overall fit factor 20 and 17, respectively). A poor seal was noted around the nose and chin and the rigidness of the straps complicated proper tightening. A fit test was not completed for the P100 filter respirator on small size standardised users due to grossly inadequate seal. Poor fit was additionally noted for regular size standardised users, overall fit factor 17 . The Self-Moldable 3D Printed respirator additionally failed to meet OSHA fit standards, overall fit factors 11 and 12, respectively, after heat moulding. The overall fit factor for the Self-Moldable 3D Printed respirator was not improved by heat moulding to users' faces, although it improved subjective user perception of fit with no subjectively noticeable air leak during normal breathing. The Multi-Part 3D Printed respirator additionally achieved poor-quality seal, overall fit factor 4 and 15 , respectively. Users noted circumferential air leak as well as potential air leak surrounding the filter screw threads. The Elastomeric respirator passed fit testing for both small and regular size standardised users, overall fit factor 110 and 108 , respectively; however, the respirator had inconsistent performance across sections of the fit test and users noted discomfort with the weight of the filter, work of breathing and strap tightness at which good fit was achieved. 
Fit Test Results

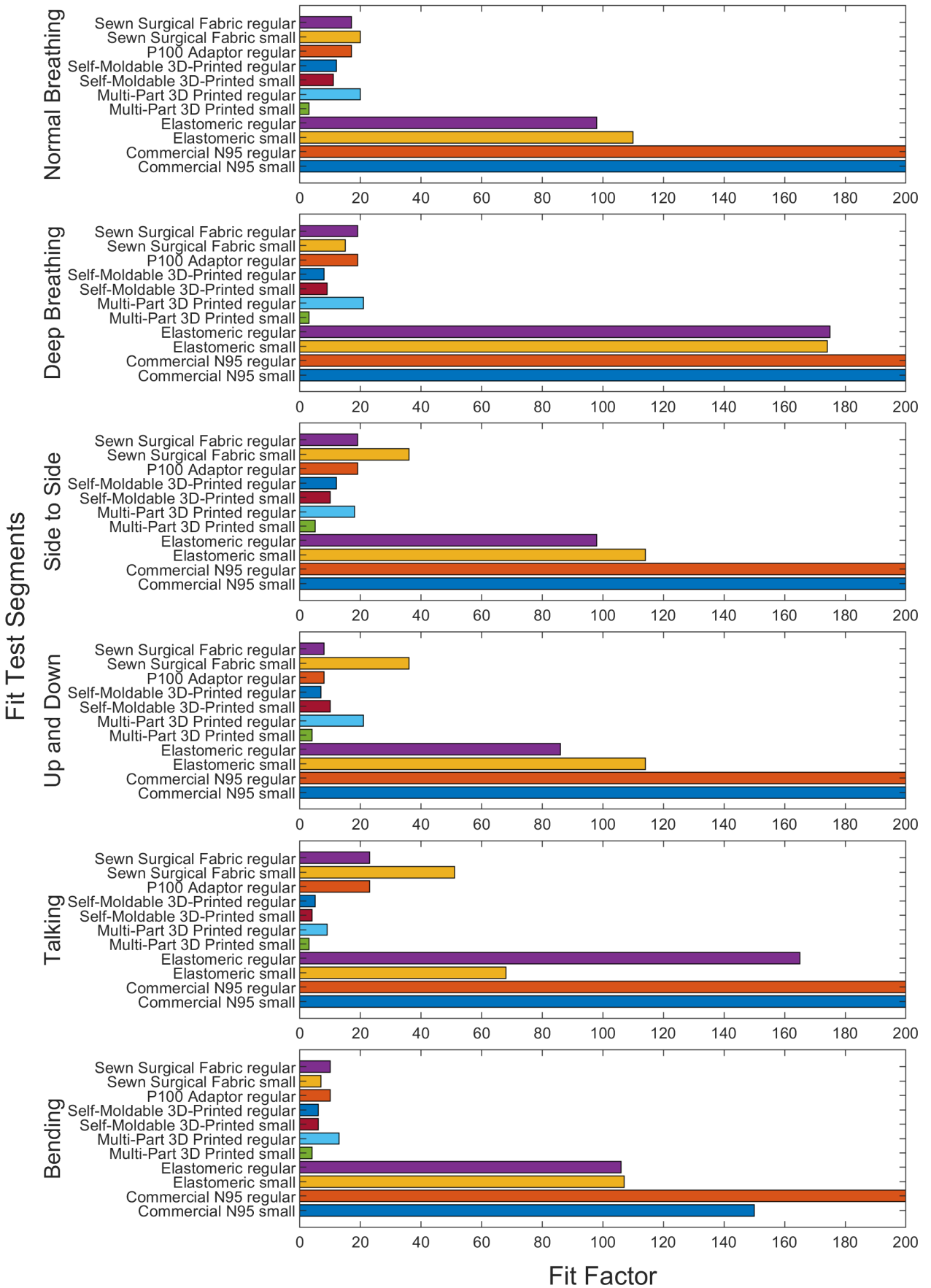

Figure 3 Fit scores across the six scored OSHA fit test sections are displayed for each respirator. An overall fit factor of 100 is required to pass testing; however, a respirator need not pass all fit testing segments as the total fit score is a weighted average of all segments. OSHA, Occupational Safety and Health Administration.

Quantitative fit factors reflect infiltration of particles through both face seal leakage and material penetration, though typical N95 FFRs have such high average filtration efficiency that poor fit is the more likely cause of failed tests (online supplemental figure 3). For improvised designs and materials, particle penetration through the filter media itself could contribute a larger fraction of particles which infiltrate the FFR, as these materials typically have poorer filtration performance. In addition, the 3D-printed designs have a lower filter media surface area, and the resulting higher air face velocities would decrease filtration performance.

\section{Material filtration and air resistance testing}

Only the commercial N95 mask material (3M VFlex Healthcare Particulate Respirator and Surgical Mask 1804, 3M, St Paul, Minnesota, USA) filtered more than $95 \%$ of $300 \mathrm{~nm}$ particles at a face velocity of $7.6 \mathrm{~cm} / \mathrm{s}$ (figure 4). In addition, the commercial N95 material had 

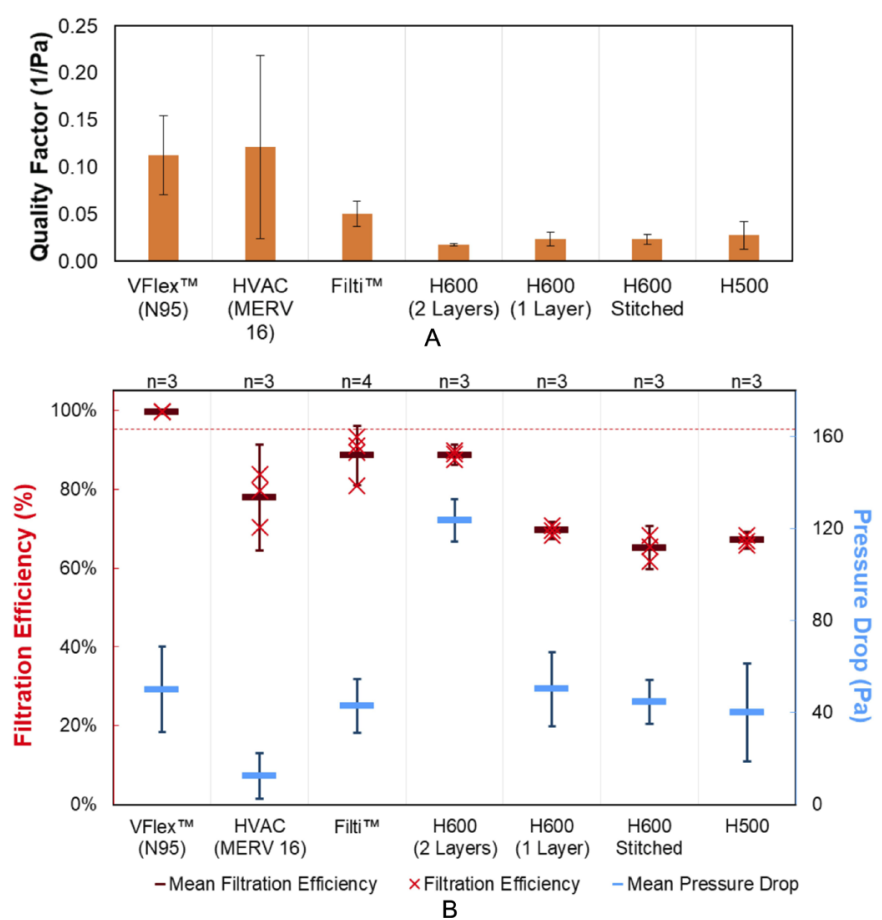

Figure 4 (A) Quality factor, (B) filtration efficiency (primary $y$-axis, red) and pressure drop (secondary y-axis, blue) observed for materials tested with an air flow face velocity of $7.6 \pm 0.1 \mathrm{~cm} / \mathrm{s}$ and $300 \mathrm{~nm}$ challenge $\mathrm{NaCl}$ particles. Error bars for filtration efficiency and pressure drop are $95 \% \mathrm{Cls}$ for mean values (represented as horizontal lines). The 95\% filtration efficiency is marked as a dashed red line.

a modest pressure drop of $50 \mathrm{~Pa}$ (95\% CI: 32 to 69$)$ at this face velocity.

The quality factor $(Q)$ enables evaluation of the trade-off between filter media filtration performance and pressure drop:

$\mathrm{Q}=\ln (1 /(1-\mathrm{E})) / \Delta \mathrm{P}$

where $\mathrm{E}$ is filtration efficiency, and $\Delta \mathrm{P}$ is pressure drop. The HVAC (MERV16) and Filti materials had higher quality factors than the sterilisation wrap materials, though their performance was more variable (a range of $12 \%$ among four punches of Filti and 13\% among three punches of the HVAC material). Two sterilisation wrap materials (H500 and H600) were tested in a variety of arrangements. As a single layer, $\mathrm{H} 500$ and $\mathrm{H} 600$ performed similarly, with slightly higher filtration efficiency ( $70 \%$ (95\% CI: $67 \%$ to $72 \%)$ ) and pressure drop ( $50 \mathrm{~Pa}(95 \%$ CI: 34 to 66)) for H600. A double layer of H600 (with the flat, less textured sides of the two layers facing inward) improved the filtration efficiency to $89 \%$ (95\% CI: $86 \%$ to $91 \%$ ), though the pressure drop increased. The filtration efficiency measurement for two layers of $\mathrm{H} 600$ sterilisation wrap was within $5 \%$ of that measured by $\mathrm{Ou}$ et $a l^{23}$ who also evaluated the impact of dry heat, steam and alcohol decontamination cycles at additional particle diameters.

To evaluate the impact of stitching Halyard material, two lines of stitches (between 6.5 and $7.0 \mathrm{~cm}$ total length) were made with a sewing machine in the centre of $47 \mathrm{~mm}$ discs of H600 material (online supplemental data document). The impact of stitching was a decrease in the filtration efficiency from the single-layer $\mathrm{H} 600$ of $70 \%$ (95\% CI: $67 \%$ to $72 \%$ ) to $65 \%$ (95\% CI: $60 \%$ to $71 \%$ ) for the stitched H600, which also had more variable performance.

A summary of the filtration efficiency and pressure drop measurements is provided in online supplemental table 1 .

\section{Breathability of improvised designs}

At the test face velocity in this study $(7.6 \mathrm{~cm} / \mathrm{s})$, none of the materials exceeded the maximum pressure drop across the filter in the NIOSH standard for N95 respirators (343 $\mathrm{Pa} \mathrm{H}_{2} \mathrm{O}$ during inhalation and $245 \mathrm{~Pa}$ during exhalation) to avoid discomfort and detrimental physiological effects. ${ }^{18}{ }^{19}$ However, the actual face velocity of a respirator undergoing this test (at a flow rate of $85 \mathrm{~L}$ / min) would depend on the surface area of filtration material (online supplemental figure 4). For fibrous filters, pressure drop and face velocity are proportional, such that we can use our measurements at a single face velocity to model the pressure drop of each material at the face velocity at which $85 \mathrm{~L} / \mathrm{min}$ of air would flow through the surface area of each design ${ }^{25}$ (online supplemental figure $5)$.

For all materials, the modelled pressure drop of the Sewn Sterilization Wrap mask is lower than the maximum standard for inhalation and exhalation. By contrast, only the HVAC material is modelled to meet this breathability standard for any of the 3D-printed designs. If the closed area of the mesh grid of the Multi-Part 3D Printed mask is not counted as available filtration surface area, then not even the HVAC material is predicted to meet the NIOSH air resistance standard when used with this design.

\section{Liquid repellency and splatter testing}

Test results and optical images of the fabric surfaces (figure 5) show that both H600 and Filti are repellent towards deionised water and milk (part A: advancing contact angles $\geq 120^{\circ}$ ), but pose potential liquid penetration points due to millimetric holes in their design. For Halyard, these holes appear sealed, whereas for Filti, the composite fabric consists of a very thin continuous layer sandwiched between two outer layers with the holes in vertical alignment. Both fabrics passed the textile absorbency test with no visible liquid penetration even after multiple minutes. Furthermore, while receding contact angles of milk on both fabrics are zero, milk stains were easily removed by wiping the surface with a wet cloth. When subject to the high-velocity milk jet (part B), however, both fabrics failed splatter testing for a single layer, as confirmed by liquid penetration (part C, bottom image 'layer 1'). When used in a double layer, $\mathrm{H} 600$ was able to prevent liquid breakthrough for all jet velocities, whereas Filti failed even as a double layer at higher impingement velocities. Whereas liquid penetration for the top layer happened uniformly at the location 


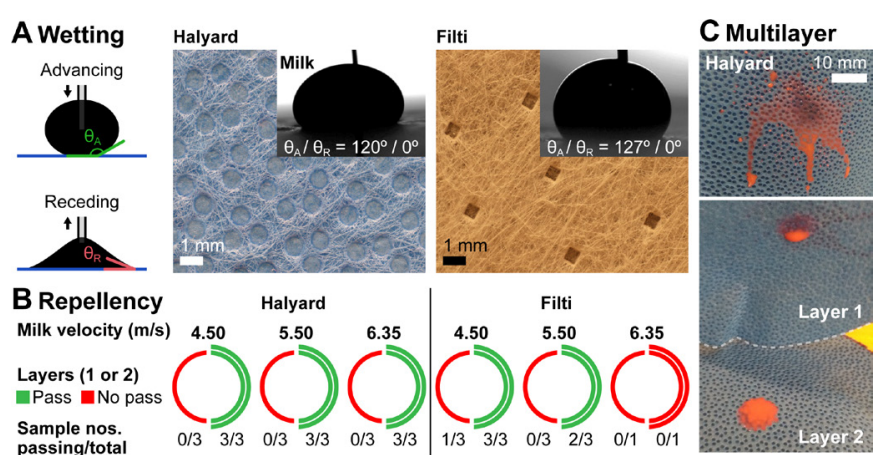

Figure 5 Fabric characterisation: wettability and splatter testing. (A) Wetting: optical images of the two tested fabrics (Halyard and Filti), along with images of milk droplets with advancing contact angles of $120^{\circ}$ and $127^{\circ}$, respectively. Visible holes pin the liquid (receding contact angles: $0^{\circ}$ ) and are a possible weak point for liquid penetration. (B) Repellency: splatter testing, that is, resistance to highvelocity liquid jet penetration (test liquid: whole milk at 4.5, 5.5 and $6.35 \mathrm{~m} / \mathrm{s}$ ), for single (left half-circle) and double (right half-circle) layers of Halyard and Filti fabrics. Red indicates repellency failure, that is, penetration of liquid through the fabric layer(s). Green indicates a passed test, if the majority of sampled fabrics did not show milk breakthrough. (C) Multilayer: optical image of the front (top) and interlayer (bottom) surfaces after liquid jet impingement. Milk (dyed with red food colour) penetrated the first layer and deposited on the underlying layer, but did not break through the second layer.

of jet impact, penetration for the bottom layer appeared predominantly through the holes in the fabric, and hence was observed more commonly for Filti and not for H600.

\section{DISCUSSION}

The COVID-19 pandemic has created significant worldwide shortages in N95 FFRs ${ }^{26-30}$ which necessitated development and publication of potential N95 respirator substitutes. ${ }^{6-11}$ Given the urgency for these N95 substitutes, safety and efficacy testing prior to their use was limited. Here we presented the results of rigorous, quantitative testing on some of the first open-source alternative N95 substitutes created to address the critical $\mathrm{N} 95$ respirator shortage at the start of the COVID-19 pandemic. In this work, a collaborative, interdisciplinary team quantitatively evaluated fit, filtration and material properties of these N95 open-source substitutes.

The focus of this paper is protocols that can be applied to test the function of improvised masks. When demonstrated on a limited number of volunteers, results revealed that most designs were not sufficiently pliable to match the contours of any of the volunteers, and therefore suggested that these designs might benefit from revision of form or materials that would improve fit prior to mass production. For the one mask that did fit a portion of the volunteers, results emphasise that careful fit testing would be required for each user of the technology. We note that the failure to fit some volunteers is not a failure of the design, in that an improvised design that performed well for individuals with only small and regular faces would still have large benefit in alleviating crisis shortages such as those encountered during the COVID-19 pandemic. In one cohort, medium and large sizes were grouped together and only represent 50 of 229 (21\%) of the cohort. ${ }^{31}$ Even with appropriate sizes, fit testing is further complicated with the shape of users' faces. ${ }^{32}$ In addition, with the same protocols required for individuals using a commercial N95 respirator in an occupational setting, fit testing could be used to verify that a particular design had adequate fit for a given individual's face.

Apart from the commercial N95 FFR, only the Elastomeric design passed quantitative fit testing. This design leverages key attributes of its commercial components, including high-quality fit of a commercial anaesthesia mask and high filtration efficiency of HEPA filter. While we did not directly test the air resistance of a single HEPA filter, the manufacturer's specification $\left(35 \mathrm{~mm} \mathrm{H}_{2} \mathrm{O}\right.$ at $\left.60 \mathrm{~L} / \mathrm{min}\right)$ indicates that it exceeds the NIOSH standard $\left(25 \mathrm{~mm} \mathrm{H}_{2} \mathrm{O}\right.$ for exhalation) even at a flow rate $(60 \mathrm{~L} / \mathrm{min})$ lower than that of the NIOSH test $(85 \mathrm{~L} / \mathrm{min}) .{ }^{33}$ Thus, a bifurcated adapter for simultaneous use of two filters is recommended for adequate breathability (modelled as $24.8 \mathrm{~mm} \mathrm{H}_{2} \mathrm{O}$ at $85 \mathrm{~L} / \mathrm{min}$ ). Although the Elastomeric design did pass, its basis off an existing commercial design may limit its implementation for mass production and distribution, as it depends on the availability of the product compared with the manufacturing capabilities of sewn masks or 3D-printed designs.

The Sewn Sterilization Wrap mask was well tolerated by users, and its larger surface area results in a modelled pressure drop (for all materials) which among the improvised proposed substitutes is most similar to the commercial N95 FFR. Both material filtration testing and quantitative fit testing indicate that its respiratory protection is not equivalent to that of an N95 FFR, though it is likely superior to that of a surgical mask (online supplemental figure 3). Two layers of sterilisation wrap also demonstrated fluid resistance in a test with a high-velocity jet of milk, though this was not strictly equivalent to the regulatory test method. Filti face mask material would not be an appropriate alternate material for improvised surgical masks or FFRs, unless combined with an additional layer that provided fluid resistance. We note that use in masks is an off-label application of sterilisation wrap.

The 3D-printed designs yielded 5 of the 6 poorest quantitative fit scores. Quantitative fit testing does not discriminate between particles which infiltrate through leaks in the face seal (or through defects) and particles which penetrate the filtration media itself. The rigidity of the 3D-printed designs compromised 
fit (as well as comfort), and the limited surface area likely exacerbated penetration through the filtration media itself. Though some reports have suggested the use of individual-specific 3D-printed masks based on their facial topography, this may not be practical for a mass production standpoint. ${ }^{3435}$ At the face velocity calculated for the N95 FFR in this study at the flow rate of a NIOSH particle filtration test, none of the alternate materials filtered more than $95 \%$ of particles. ${ }^{22}$ Since their lower surface area would result in a higher face velocity in an NIOSH particle filtration test, the 3D-printed masks would likely have lower filtration efficiency than reported here for these materials. Only the HVAC material was modelled to have low enough air resistance for the 3D-printed designs at these high face velocities, such that we recommend pressure drop measurements of specific filter media proposed for these designs. More specifically, measuring or modelling air resistance at the face velocity which would be encountered in an NIOSH test (at $85 \mathrm{~L} / \mathrm{min}$ ) enables a direct comparison of an improvised design with the N95 standard.

Even without direct filtration testing of full prototypes (which is experimentally more demanding), we demonstrate how quantitative fit testing and material filtration testing can be combined to screen proposed improvised designs together with consideration of air and fluid resistance. These results point to a fundamental need to improve facial fit in future respirator designs, and even more acutely, to an ongoing need during this pandemic for end users to be equipped and educated for some measure of fit testing. In addition, evaluating designs at the conditions of regulatory test methods (eg, appropriate face velocity for filtration and air resistance) enables direct comparison to the performance expected of an N95 FFR.

There are several limitations to the present study. Our working group identified designs based on designs in the published literature, designs in the mainstream media and designs that were proposed to the Washington University hospital system. Although these designs were by no means exhaustive and their selection represented a degree of media bias, they nevertheless represented a sufficiently diverse sampling of improvisation and innovation to illustrate the need to evaluate efficacy and to demonstrate the protocols that are the focus of this paper. Although this study does not evaluate improvised respirator designs as a category (in which case sampling bias would be of concern), we did not attempt to test all of the large number of potential N95 respirator substitutes. The improvised respirator-proposed substitutes were reproduced to the best understanding of posted instructions; however, the tested designs may not reflect interval improvements. To demonstrate these protocols, fit testing was carried out with a limited number of individuals who passed fit testing of analogous small-sized and regular-sized N95 respirators. For designs such as the elastomeric design, which was the only one to passed the fit test for any of the seven volunteers, additional testing would be warranted for each individual who used this design. Although this limited testing was not designed to develop statistically significant datasets on the proportion of the population that might be able to use each mask design effectively, it did serve to both demonstrate repeatable protocols and to establish limitations of the designs that were not sufficiently pliable to pass fit testing for any of the volunteers.

While filtration testing of material patches at relevant conditions can inform material selection for further development, filtration tests of a mask prototype in its complete form are necessary for evaluation against N95 NIOSH standards, and we continue to develop in-house capacity for these tests. A complication is that the face velocity of a mask depends on a user's minute ventilation, respiratory rate, inspiratory time and the mask surface area, complicating comparison of masks and protocol standardisation. Whole milk was used to test the splatter resistance of the fabrics, as artificial blood was not readily accessible. While the measured surface tension is within the range of surface tension of typical body fluids and blood at body temperature, ${ }^{24}{ }^{36}$ it is slightly higher than that of synthetic blood as prescribed by F1862, which could result in favourable test results, as fluids with lower surface tension are known to wet surfaces more easily. ${ }^{37}$

The potential N95 respirator substitutes tested here were attempts to meet immediate needs of the COVID-19 pandemic frontline. However, our data indicate the majority of these proposed substitutes do not have equivalent respiratory protection and breathability to an N95 FFR. The majority of masks tested revealed inherent design issues such as inadequate filtration capabilities of the base materials and poor ergonomic facial fit to a variety of facial shapes and sizes. Our experience has highlighted the importance for institutions to be equipped and educated to perform appropriate qualitative and quantitative testing prior to novel mask implementation. This study reveals that rapid creation of an improvised respirator with N95 performance using readily available materials and simple manufacturing methods is extremely challenging, and consequently there is an emergent need for in-house testing platforms to better understand the degree to which protection is being provided. Healthcare professionals requiring this high level of respiratory protection should be cautious of claims associated with improvised respirators when suggested as N95 replacements without quantitative evaluation.

\section{Author affiliations}

${ }^{1}$ School of Medicine Mallinckrodt Institute of Radiology, Washington University, Saint Louis, Missouri, USA

${ }^{2}$ Department of Energy, Environmental and Chemical Engineering, Washington University in St Louis, St Louis, Missouri, USA

${ }^{3}$ Department of Mechanical Engineering \& Materials Science, Washington University in St Louis, St Louis, Missouri, USA

${ }^{4}$ School of Medicine, Washington University in St Louis, St Louis, Missouri, USA 
${ }^{5}$ Sam Fox School of Design and Visual Arts, Washington University in St Louis, St Louis, Missouri, USA

${ }^{6}$ Department of Anesthesiology, Washington University in St Louis, St Louis, Missouri, USA

${ }^{7}$ Department of Environmental Health \& Safety, Washington University in St Louis, St Louis, Missouri, USA

${ }^{8}$ Division of Infectious Diseases, Washington University in St Louis, St Louis, Missouri, USA

${ }^{9}$ Department of Anesthesiology, Memorial Hospital Belleville, St Louis, Missouri, USA

${ }^{10}$ Department of Otolaryngology-Head \& Neck Surgery, Washington University in St Louis, St Louis, Missouri, USA

${ }^{11}$ Division of Comparative Medicine, Washington University in St Louis, St Louis, Missouri, USA

${ }^{12}$ NSF Science and Technology Center for Engineering Mechanobiology, Washington University in St. Louis, St Louis, Missouri, USA

${ }^{13}$ Bioinspired Engineering and Biomechanics Center, School of Life Sciences and Technology, Xi'an Jiaotong University, China, Xi'an, China

\section{Twitter David H Ballard @DavidBallardMD}

Acknowledgements The Washington University N95 design task force for their support and facilitating testing.

Contributors Concept and experimental design—DHB, AJD, BMK, PBW, JMM, BAB, JTB, NS, PKW, PB, RA, GG, BJW and KM. Experimental studies-DHB, AJD, BMK, PBW, JMM, ARS, MR-S, BAB, JM, CG, JH, BK, UJ, SC, DD, BM, CM, JTB, NS, PKW, BJW and KM. Data collection-DHB, AJD, BMK, PBW, JMM, ARS, MR-S, BAB, JM, CG, JH, BK, UJ, SC, DD, BM, JTB, NS, PKW, BJW and KM. First manuscript draftDHB, AJD, BMK, PBW, JMM, ARS, MR-S, BAB, JM, CG, JH, BK, SS, SL, SC, DD, NS, RA, GG, BJW and KM. Critical revision-DHB, AJD, BMK, PBW, JMM, ARS, BAB, BK, JTB, PKW, NS, RA, GG, BJW and KM. Approval of final manuscript-all authors.

Funding This work was supported in part by a National Science Foundation Graduate Research Fellowship (DGE-1745038) and International Anesthesiology Research Society Mentored Research Award. BAB and KM receive salary support from International Anesthesiology Research Society Mentored Research Award. AJD was supported by a National Science Foundation Graduate Research Fellowship (DGE-1745038).

Disclaimer Any opinions, findings, and conclusions or recommendations expressed in this material are those of the authors and do not necessarily reflect the views of the National Science Foundation.

Competing interests None declared.

Patient consent for publication Not required.

Ethics approval The Washington University Human Research Protection Office determined that this study (which included fit testing of respirator designs by adult volunteers without collection of personal data) was designated non-human subjects research and was exempt from Institutional Review Board oversight (reference ID \#202003144).

Provenance and peer review Not commissioned; externally peer reviewed.

Data availability statement Data are available upon reasonable request. Data not presented in the present manuscript may be provided by the corresponding author upon a reasonable request.

Supplemental material This content has been supplied by the author(s). It has not been vetted by BMJ Publishing Group Limited (BMJ) and may not have been peer-reviewed. Any opinions or recommendations discussed are solely those of the author(s) and are not endorsed by BMJ. BMJ disclaims all liability and responsibility arising from any reliance placed on the content. Where the content includes any translated material, BMJ does not warrant the accuracy and reliability of the translations (including but not limited to local regulations, clinical guidelines, terminology, drug names and drug dosages), and is not responsible for any error and/or omissions arising from translation and adaptation or otherwise.

Open access This is an open access article distributed in accordance with the Creative Commons Attribution Non Commercial (CC BY-NC 4.0) license, which permits others to distribute, remix, adapt, build upon this work non-commercially, and license their derivative works on different terms, provided the original work is properly cited, appropriate credit is given, any changes made indicated, and the use is non-commercial. See: http://creativecommons.org/licenses/by-nc/4.0/.

\section{ORCID iD}

David H Ballard http://orcid.org/0000-0001-7470-3364
REFERENCES

1 World Health Organization. Rational use of personal protective equipment for coronavirus disease 2019 (COVID-19) - interim guidance, 2020. Available: https://apps.who.int/iris/bitstream/handle/ 10665/331215/WHO-2019-nCov-IPCPPE_use-2020.1-eng.pdf [Accessed 13 Apr 2021].

2 "Protecting healthcare wrkers during the COVID-19 pandemic: a survey of infection preventionists March 27, 2020." Association for professionals in infection control and epidemiology. Available: https://apic.org/wp-content/uploads/2020/03/Protecting-HealthcareWorkers-Survey_Report_3_26_20_Final.pdf [Accessed 13 Apr 2021].

3 Provenzano D, Rao YJ, Mitic K. Rapid prototyping of reusable 3D-printed N95 equivalent respirators at the George Washington University. Biomed Chem Eng 2020:2020030444.

4 Liu DCY, Koo TH, Wong JKK, et al. Adapting re-usable elastomeric respirators to utilise anaesthesia circuit filters using a 3D-printed adaptor - a potential alternative to address N95 shortages during the COVID-19 pandemic. Anaesthesia 2020;75:1022-7.

5 Elkington P, Dickinson A, Mavrogordato M. A personal respirator specification for health-care workers treating COVID-19 (PeRSo).

6 NIH 3D Print Exchange. COVID-19 supply chain response collection. Available: https://3dprint.nih.gov/collections/covid-19-response/ search [Accessed 13 Apr 2021].

7 University of Florida College of Medicine Department of Anesthesiology. Mask alternative. Available: https://anest.ufl.edu/ clinical-divisions/mask-alternative/\#prototype2 [Accessed $13 \mathrm{Apr}$ 2021].

8 Barrow Neurological Institute. 3D printed N95 replacement mask. Available: https://www.barrowneuro.org/get-to-know-barrow/barrowinnovation-center-2/3d-printed-n95-mask/ [Accessed 13 Apr 2021].

9 Make the Masks. The montana mask. Available: https://www. makethemasks.com/

10 River City Labs. The "MalaMask" project (N95 alternative filter). Available: https://wiki.rivercitylabs.space/covid-19/3d-printed-masks [Accessed 13 Apr 2021].

11 Boston Children's Hospital. Surgical innovation fellowship. Available: http://www.childrenshospital.org/research/departments-divisionsprograms/departments/surgery/surgical-innovation-fellowship [Accessed 13 Apr 2021].

12 Radonovich LJ, Simberkoff MS, Bessesen MT, et al. N95 respirators vs medical masks for preventing influenza among health care personnel: a randomized clinical trial. JAMA 2019;322:824-33.

13 Janssen L, Ettinger $\mathrm{H}$, Graham $\mathrm{S}$, et al. The use of respirators to reduce inhalation of airborne biological agents. J Occup Environ Hyg 2013;10:D97-103.

14 N95 decontamination \& reuse method decision matrix, N95DECON. Available: https://static1.squarespace.com/static/5e8126f89327941b 9453eeef/t/5ffe0c426639ea757c0ee5e7/1610484802173/20200816 N95_Decontamination_Reuse_Comparison_Matrix.pdf [Accessed 13 Apr 2021].

15 Viscusi DJ, Bergman MS, Eimer BC, et al. Evaluation of five decontamination methods for filtering facepiece respirators. Ann Occup Hyg 2009;53:815-27.

16 Boškoski I, Gallo C, Wallace MB, et al. COVID-19 pandemic and personal protective equipment shortage: protective efficacy comparing masks and scientific methods for respirator reuse. Gastrointest Endosc 2020;92:519-23.

17 United States Department of Labor. Appendix A to §1910.134Fit testing procedures (mandatory). Available: https://www.osha. gov/laws-regs/regulations/standardnumber/1910/1910.134AppA [Accessed 13 Apr 2021].

18 Balazy A, Toivola M, Reponen T, et al. Manikin-based performance evaluation of N95 filtering-facepiece respirators challenged with nanoparticles. Ann Occup Hyg 2006;50:259-69.

19 Martines RB, Ritter JM, Matkovic E, et al. Pathology and pathogenesis of SARS-CoV-2 associated with fatal coronavirus disease, United States. Emerg Infect Dis 2020;26:2005-15.

20 Johnson GR, Morawska L, Ristovski ZD, et al. Modality of human expired aerosol size distributions. J Aerosol Sci 2011;42:839-51.

21 Cornell Law School Legal Information Institute. Airflow resistance test. Available: https://www.law.cornell.edu/cfr/text/42/84.172 [Accessed 13 Apr 2021].

22 Chen V, Long K, Woodburn EV. When weighing universal precautions, filtration efficiency is not universal. J Hosp Infect 2020.

$23 \mathrm{Ou}$ Q, Pei C, Chan Kim S, et al. Evaluation of decontamination methods for commercial and alternative respirator and mask materials - view from filtration aspect. J Aerosol Sci 2020;150:105609.

24 Stalder AF, Kulik G, Sage D, et al. A snake-based approach to accurate determination of both contact points and contact angles. Colloid Surface A 2006;286:92-103. 
25 Hinds WC. Aerosol technology. 2nd edn. Hoboken: Wiley, 1999.

26 van Doremalen N, Bushmaker T, Morris DH, et al. Aerosol and surface stability of SARS-CoV-2 as compared with SARS-CoV-1. N Engl J Med 2020;382:1564-7.

27 Bauchner H, Fontanarosa PB, Livingston EH. Conserving supply of personal protective equipment-A call for ideas. JAMA 2020;323:1911.

28 Wong SCY, Kwong RT-S, Wu TC, et al. Risk of nosocomial transmission of coronavirus disease 2019: an experience in a general ward setting in Hong Kong. J Hosp Infect 2020;105:119-27.

29 Lu J, Yang Z, et al. COVID-19 outbreak associated with air conditioning in restaurant, Guangzhou, China, 2020. Emerg Infect Dis 2020;26:2789-91.

30 Santarpia JL, Rivera DN, Herrera V. Transmission potential of SARSCoV-2 in viral shedding observed at the University of Nebraska medical center. Infect Dis 2020.

31 Bergman M, Zhuang Z, Brochu E, et al. Fit assessment of N95 filtering-facepiece respirators in the U.S. centers for disease control and prevention strategic national stockpile. J Int Soc Respir Prot 2015;32:50-64.
32 ASTM F3407-20, standard test method for respirator fit capability for negative-pressure half- facepiece particulate respirators. ASTM international, West Conshohocken, PA, 2020. Available: http://www. astm.org/cgi-bin/resolver.cgi?F3407 [Accessed 13 Apr 2021].

33 Ultipor® 25 anesthesia filter with monitoring port. Available: https:// shop.pall.com/us/en/products/zidBB25AB?CategoryName=\& CatalogID=\&tracking=searchterm [Accessed 13 Apr 2021].

34 Swennen GRJ, Pottel L, Haers PE. Custom-made 3D-printed face masks in case of pandemic crisis situations with a lack of commercially available FFP2/3 masks. Int J Oral Maxillofac Surg 2020;49:673-7.

35 Scott AR, Hu J, Gan C, et al. Safety concerns for facial topography customized 3D-printed N95 filtering face-piece respirator produced for the COVID-19 pandemic: initial step is respiratory fit testing. Int $J$ Oral Maxillofac Surg 2020;49:1660-1.

36 AATCC. AATCC TM79-2010e2, 2018. Available: https://members. aatcc.org/store/tm79/499/ [Accessed 13 Apr 2021].

37 Daerr A, Mogne A. Pendent_drop: an imageJ plugin to measure the surface tension from an image of a pendent drop. J Open Res Softw 2016;4:e3. 